\title{
BMJ Open Quality Human factors in escalating acute ward care: a qualitative evidence synthesis
}

\author{
Jody Ede (10 , ${ }^{1,2}$ Tatjana Petrinic, ${ }^{3}$ Verity Westgate, ${ }^{4}$ Julie Darbyshire, ${ }^{4}$ \\ Ruth Endacott (D) ,2,5 Peter J Watkinson (D) ${ }^{4}$
}

To cite: Ede J, Petrinic T, Westgate $\mathrm{V}$, et al. Human factors in escalating acute ward care: a qualitative evidence synthesis. BMJ Open Quality 2021;10:e001145. doi:10.1136/ bmjoq-2020-001145

- Additional material is published online only. To view, please visit the journal online (http://dx.doi.org/10.1136/ bmjoq-2020-001145).

Received 17 August 2020 Revised 7 January 2021 Accepted 4 February 2021
Check for updates

(c) Author(s) (or their employer(s)) 2021. Re-use permitted under CC BY. Published by BMJ.

${ }^{1}$ Adult Intensive Care Unit, Oxford University Hospitals NHS Foundation Trust, Oxford, UK ${ }^{2}$ Plymouth University, Plymouth, UK

${ }^{3}$ Bodleian Health Care Libraries, University of Oxford, Oxford, UK ${ }^{4}$ Nuffield Department of Clinical Neurosciences, University of Oxford, Oxford, UK

${ }^{5}$ School of Nursing \& Midwifery, Monash University, Clayton, Victoria, Australia

Correspondence to

Mrs Jody Ede;

jody.ede@ouh.nhs.uk

\section{ABSTRACT}

Background Identifying how human factors affect clinical staff recognition and managment of the deteriorating ward patient may inform process improvements. We systematically reviewed the literature to identify (1) how human factors affect ward care escalation (2) gaps in the current literature and (3) critique literature methodologies. Methods We undertook a Qualitative Evidence Synthesis of care escalation studies. We searched MEDLINE, EMBASE and CINHAL from inception to September 2019. We used the Critical Appraisal Skills Programme and the Grading of Recommendations Assessment-Development and Evaluation and Confidence in Evidence from Reviews of Qualitative Research tool to assess study quality.

Results Our search identified 24 studies meeting the inclusion criteria. Confidence in findings was moderate (20 studies) to high (4 studies). In 16 studies, the ability to recognise changes in the patient's condition (soft signals), including skin colour/temperature, respiratory pattern, blood loss, personality change, patient complaint and fatigue, improved the ability to escalate patients. Soft signals were detected through patient assessment (looking/listening/feeling) and not Early Warning Scores (eight studies). In contrast, 13 studies found a high workload and low staffing levels reduced staff's ability to detect patient deterioration and escalate care. In eight studies quantifiable deterioration evidence (Early Warning Scores) facilitated escalation communication, particularly when referrer/referee were unfamiliar. Conversely, escalating concerning non-triggering patients was challenging but achieved by some clinical staff (three studies). Team decision making facilitated the clinical escalation (six studies).

Conclusions Early Warning Scores have clinical benefits but can sometimes impede escalation in patients not meeting the threshold. Staff use other factors (soft signals) not captured in Early Warning Scores to escalate care. The literature supports strategies that improve the escalation process such as good patient assessment skills.

PROSPERO registration number CRD42018104745.

\section{INTRODUCTION}

\section{Failure to rescue}

'Failure to rescue' (FTR), defined as mortality following complications during a hospital admission, ${ }^{1}$ is common. ${ }^{2}$ At least 11 000 hospital patients each year suffer preventable deaths ${ }^{3}$ though other sources believe this number to be higher. ${ }^{4}$ It is also recognised that patients who die following a cardiac arrest are likely to have preceding warning signs that are not adequately managed. ${ }^{5}$ Though differences between hospital complication rates are small, patients can be three times more likely to die from complications depending on which hospital they are in. ${ }^{2}$ Poor surveillance of these patients can be linked to inadequate monitoring of abnormal vital signs, poor fluid balance management or diagnostic errors. ${ }^{36}$ Reports to the National Reporting and Learning System deomstrate that $7 \%$ were related to a failure to act or recognise patient deterioration. ${ }^{7}$

\section{Escalation of care}

Avoiding FTR requires successful escalation of care ${ }^{8}$ whereby patients' deteriorations are detected, communicated and acted on. ${ }^{8}{ }^{9}$ Escalation interventions focus primarily on specialist clinical teams such as Critical Care Outreach or Rapid Response Teams (RRT). ${ }^{10}$ These teams aim to target improvements to the initial detection and ward management of patient deterioration. ${ }^{11}$ Other interventions target communication breakdowns. ${ }^{12}$

Human factors (HF) identified to positively or negatively affect care escalation include situational awareness, team working, communication, safety culture, workload, clinical experience, negative emotions and leadership. ${ }^{6}{ }^{13-15}$ However, research has historically focused on outcomes. ${ }^{8}$ The aims of this qualitative evidence synthesis (QES) are to identify (1) how HF affect ward care escalation (2) gaps in the current literature and (3) critique literature methodologies.

\section{METHODS}

The Preferred Reporting Items for Systematic Reviews and Meta-Analyses (PRISMA) guideline was adhered to ${ }^{16}$ (see online supplemental file 1). We undertook a QES of the literature exploring escalation of care. The research question was developed by the two authors (JE and VW) using the Population, Interest and Context framework. ${ }^{17} \mathrm{~A}$ full protocol has been published in a peer-reviewed journal. ${ }^{18}$ 
The search strategy was assisted by a specialist librarian (TP). Searches were performed on three databases, MEDLINE, EMBASE and CINHAL. Dates searched were from database inception to September 2019. Medical Subject Headings terms were used and searched as free text (full search strategy is included in online supplemental file 2). Reference lists of all eligible studies were also checked, and incidental references included from these.

\section{Eligibility criteria}

This evidence synthesis includes qualitative studies reporting primary data. No limits on publication date or country were applied. We included studies that explored how HF affect FTR and care escalation from staff, patients or relative's perspective. Qualitative methods include (but are not limited to) ethnography, interviews, focus groups and HF methods. We defined HF as any human interaction affecting teamwork, tasks, equipment, workspace, culture or organisation. ${ }^{19}$ Data analysis included, but has not been not limited to, thematic analysis, grounded theory and discourse analysis.

\section{Inclusion}

- Qualitative studies reporting primary data.

- Qualitative studies exploring how HF affect escalation of care of the in-hospital patient population.

- Studies employing qualitative data collection methods, for example, semistructured interviews, focus groups or observations.

- Observational studies relating to FTR or care escalation.

- Adult population.

Exclusion

- Systematic or literature reviews.

- Correspondence and short communications.

- Simulation studies.

- Studies written in any language other than English.

- Studies in the emergency department and maternity.

Eligible studies were entered into Covidence systematic review software (Veritas Health Innovation, Melbourne, Australia. Available at www.covidence.org) and deduplicated. Study screening and selection was undertaken by two reviewers independently. The titles and abstracts were screened against the eligibility criteria. Disagreements between reviewers were resolved by third person mediation. Reasons for excluding studies were noted.

\section{Quality assessment and confidence in synthesised} findings (Critical Appraisal Skills Programme and Grading of Recommendations Assessment, Development and Evaluation-CERQual)

Two researchers (JE and VW) reached a consensus regarding which study quality assessment tools to use during the review. Two different quality assessments were conducted on all studies by both researchers. The Critical Appraisal Skills Programme (CASP) qualitative checklist was used to assess papers for credibility, confirmability, dependability and transferability. ${ }^{20}$ This comprehensive framework tool is commonly used in qualitative study assessment. ${ }^{21} 22$

We assessed confidence in synthesised findings using the Grading of Recommendations Assessment-Development and Evaluation and Confidence in Evidence from Reviews of Qualitative Research (GRADE-CERQual) Criteria and associated guidance publications. ${ }^{23-27}$ The four-stage assessment (methodological limitations, coherence, adequacy of data and relevance) examines each synthesised finding for confidence by critiquing contributing study rigour. ${ }^{28}$ The output of this evaluation is a Summary of Qualitative Findings table detailing themes and papers contributing to this theme. This table promotes transparency in the synthesis methods. Themes from the data analysis are presented in order of highest to lowest confidence according to the GRADE-CERQual assessment.

\section{Analysis}

We undertook a thematic synthesis ${ }^{22}$ using Thomas and Harden's framework to map how HF affect escalation of care. ${ }^{29}$ This is a three-stage process. Initially, study findings are coded, these codes are then categorised into descriptive themes and finally these descriptive themes are categorised into analytical themes. ${ }^{30}$ Stage 1 involves line-by-line coding of data, where each sentence is allocated a code. Stage 2 involves categorising each coded sentence into descriptive, broader themes. The final stage involves generating analytical themes, or 'going beyond' the findings of the initial study, which relate to the fixed or emerging research question (see table 1 for definitions of analytical themes). This framework supports data extraction from anywhere within the paper and is not confined to the results alone.

Data extraction tools were developed and piloted before the review took place to ensure consistency of data extraction. Study data were entered into an Excel spreadsheet (Windows, 2019. Microsoft Office) and study themes were analysed using NVivo software (NVivo qualitative data analysis Software; QSR International, V.10, 2014).

\section{Patient and public involvement}

Patient representative (TD) reviewed the original published protocol and aims of the review were discussed and deemed of patient importance.

\section{RESULTS}

The search identified 2404 papers which met the initial search criteria (refer to online supplemental file 3 for PRISMA diagram). After duplicates were removed, 1651 articles were screened. 1627 were excluded based on methodology, subject of interest or incorrect population. This resulted in 24 papers meeting the inclusion criteria and being reviewed in full. A full description of synthesised study characteristics are presented in table 2. 
Table 1 Definitions of analytical themes

\begin{tabular}{|c|c|}
\hline Analytical theme & Definitions and references \\
\hline Information packaging & $\begin{array}{l}\text { The use of quantifiable evidence of deterioration (such as vital signs) to initiate escalation of } \\
\text { care. }^{1533344041}\end{array}$ \\
\hline Situational awareness & The comprehension of clinical elements and projection of their status in the future. ${ }^{73}$ \\
\hline Team functioning & $\begin{array}{l}\text { Fragmented team-working with sequential rather than concurrent task completion and poor } \\
\text { relationships. } 353641-454748\end{array}$ \\
\hline Decision making & $\begin{array}{l}\text { Clinical reasoning surrounding detection, communication and management of escalation of } \\
\text { care. }\end{array}$ \\
\hline Clinical experience & 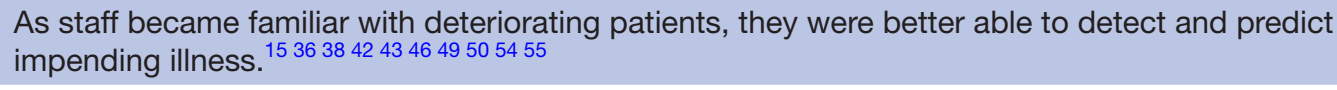 \\
\hline
\end{tabular}

\section{Quality assessment results}

\section{Critical Appraisal Skills Programme}

Studies were assessed to be of moderate to high quality and no studies were excluded based on this assessment (table 3). Two studies ${ }^{31}$ used surveys to understand nurses' perceptions of caring for deteriorating patients and were scored poorly for choice of methodology. These studies were still included as open ended freetext questions were used and it was felt that this could still contribute to answering the research question, while acknowledging data from these studies are unlikely to be rich and is therefore a limitation of the method.

\section{Grading of Recommendations Assessment, Development and Evaluation-CERQual}

Following the CASP assessment all studies were evaluated against the GRADE-CERQual criteria. A Summary of Qualitative Findings table (table 4 ) is presented which promotes transparency in this synthesis' findings and methods. The table includes documented rationale for grading judgements.

\section{THEME RESULTS}

Themes presented are ranked from the highest to lowest confidence in synthesised findings. Data extracted mostly related to organisational and patient assessment factors affecting escalation of care. Organisational factors could be classified into Information Packaging and Communication Credibility, Flattened Hierarchy, Workload, Staffing and Situational Awareness and Team Functioning. We found patient assessment Themes of 'Soft Signals of Patient Deterioration' and Early Warning Scores (EWS), Decision Making and Clinical Assessment Skills and Experience.

\section{Information packaging and communication credibility (high} GRADE-CERQual evidence)

Eight studies identified that information packaging during escalation of care was a facilitator to success. ${ }^{1533-39}$
Packaging involved using quantifiable evidence of patient deterioration such as vital signs ${ }^{15} 33344041$ to initiate escalation of care. This removed ambiguity, ${ }^{33}$ provided numerical evidence of deterioration ${ }^{15}$ and was a common language ${ }^{34}$ for clinical staff. This was particularly important when staff were unfamiliar to each other. ${ }^{34}$ Conversely, staff felt communication credibility was questioned when referrals were made using non-medical language ${ }^{33}$ or delivered in an unsystematic way. ${ }^{34}$ This made an escalation referral difficult to understand and prioritise, with medics often having to question further to gain more information to facilitate decision making. ${ }^{33}$

\section{Flattened hierarchy (high GRADE-CERQual evidence)}

A common organisational facilitator to escalation of care was a flattened hierarchy meaning that escalation is accepted from anyone to anyone. ${ }^{15313541-45}$ This created a confidence in staff to raise concerns regarding a patient's clinical condition, opening channels of communication. Staff also felt that electronic vital signs systems increased the accountability of patient illness ${ }^{32} 354246$ with acutely unwell patients being everyone's responsibility. However, it was also acknowledged identifying who is accountable for an unwell patient was sometime a challenging. Synthesised studies demonstrated instances of lack of deteriorating patient ownership ${ }^{32}$ or passing on of the problem ${ }^{34}$ by clinical staff to another team or colleague.

\section{Workload, staffing and situational awareness (high GRADE- CERQual evidence)}

Several studies described resources as a significant factor affecting care escalation. Three studies identified a lack of skilled staff as limiting the ability to escalate the deteriorating patient. ${ }^{15} 3547$ During high workload or low staffing periods staff felt their awareness of patient deterioration reduced due to sensory overload and suboptimal monitoring due to competing demands. ${ }^{15} 32343641424547-52$ Staff believed continuity of care improved situational 


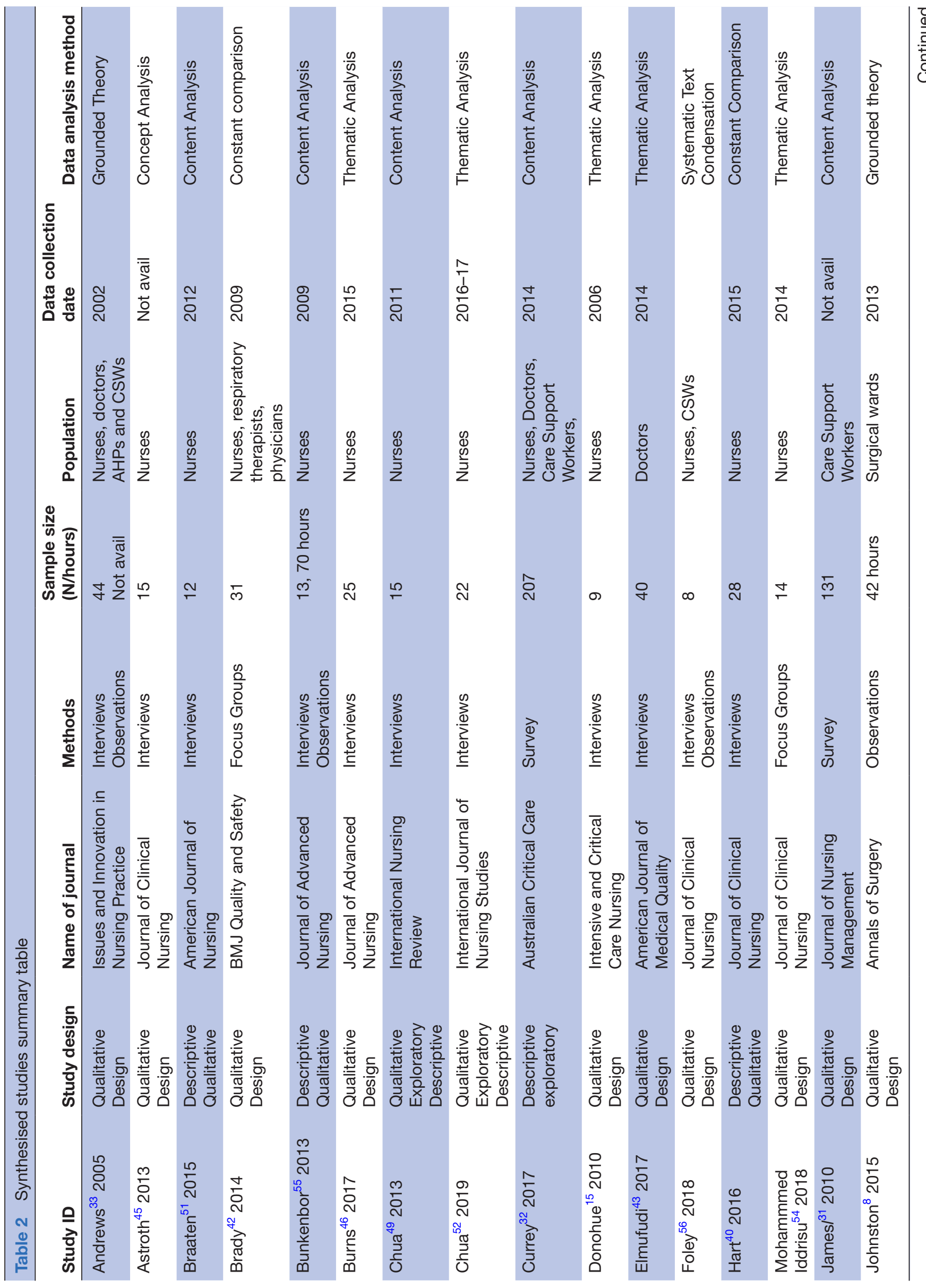




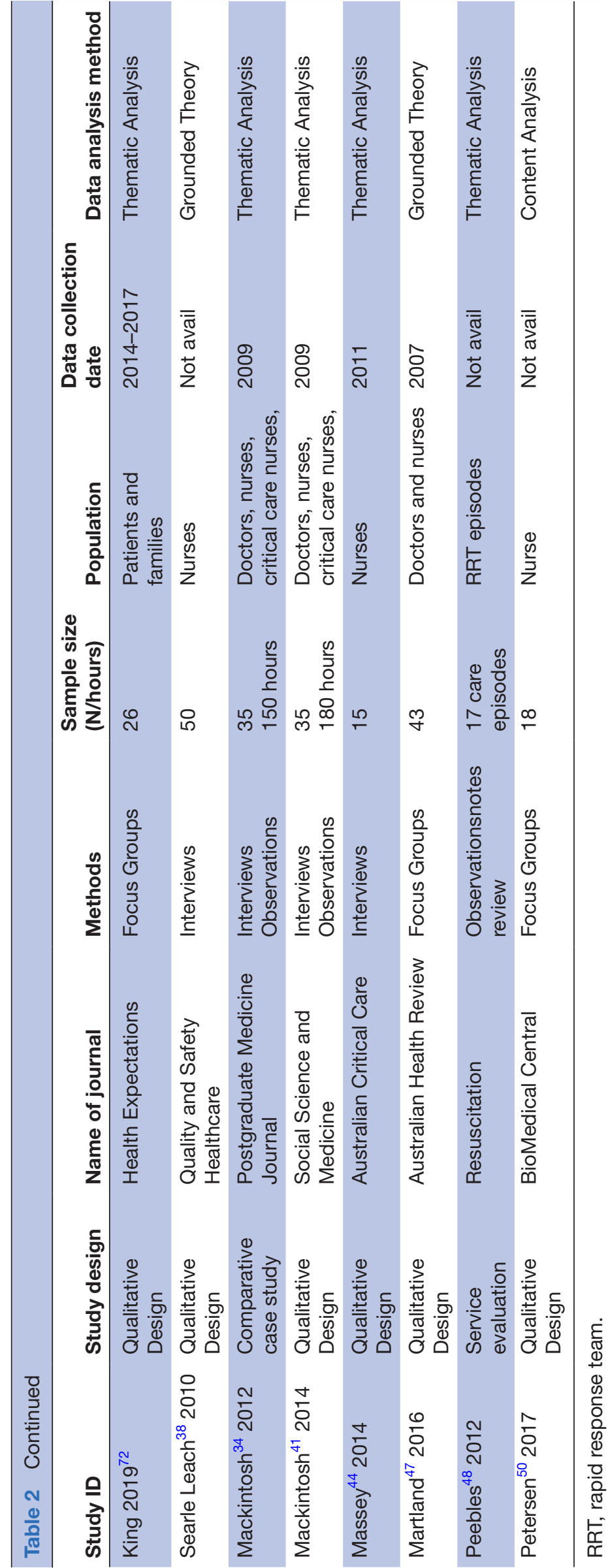




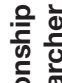

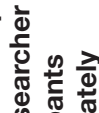

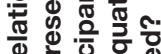

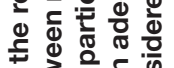

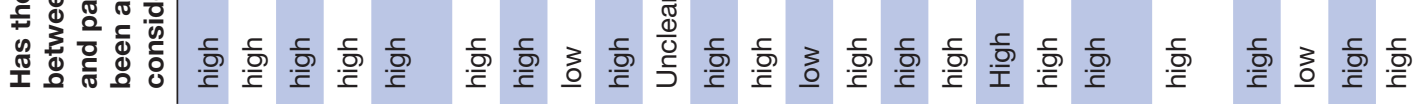

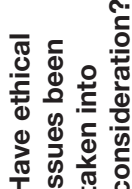

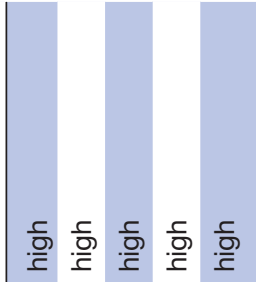

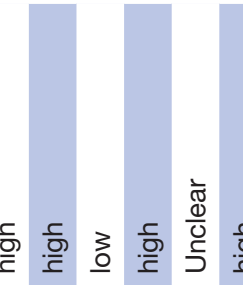

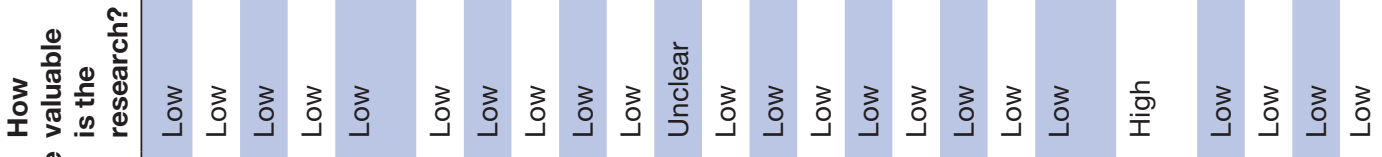

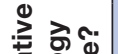

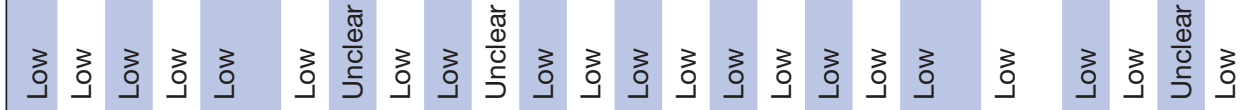
능 흔

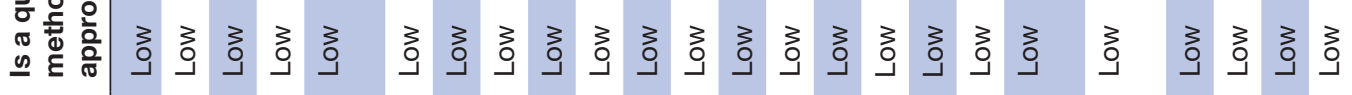

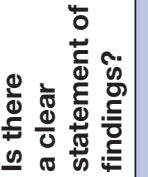

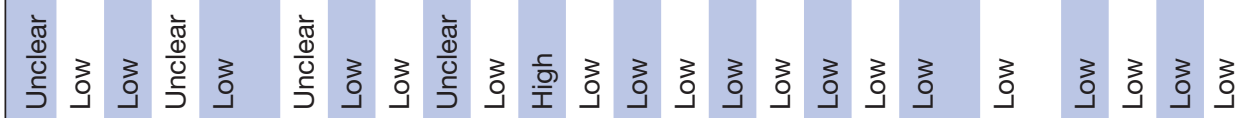

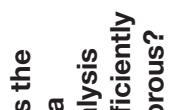

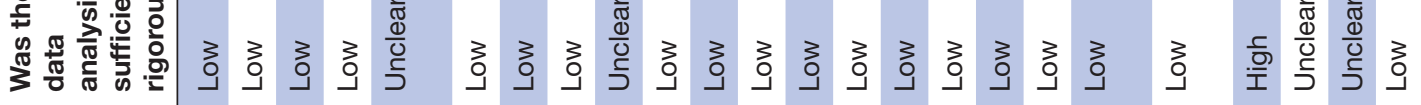

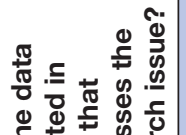

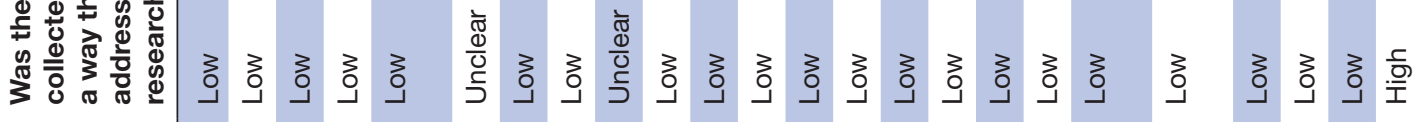
$\stackrel{0}{\stackrel{5}{5}}$ 苛 $\quad$

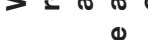

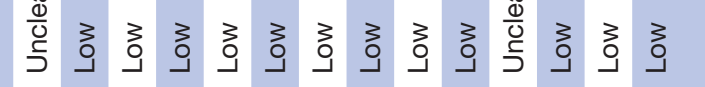

3.

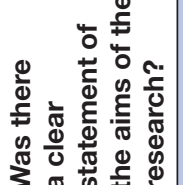

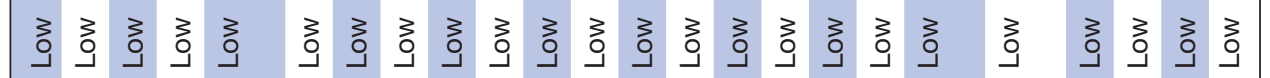

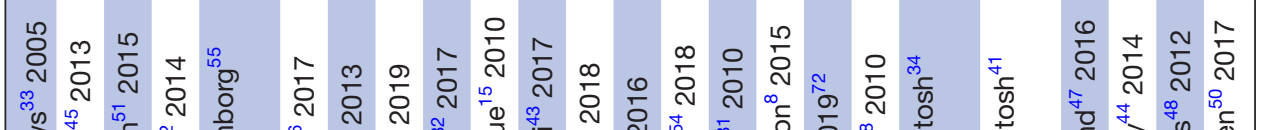




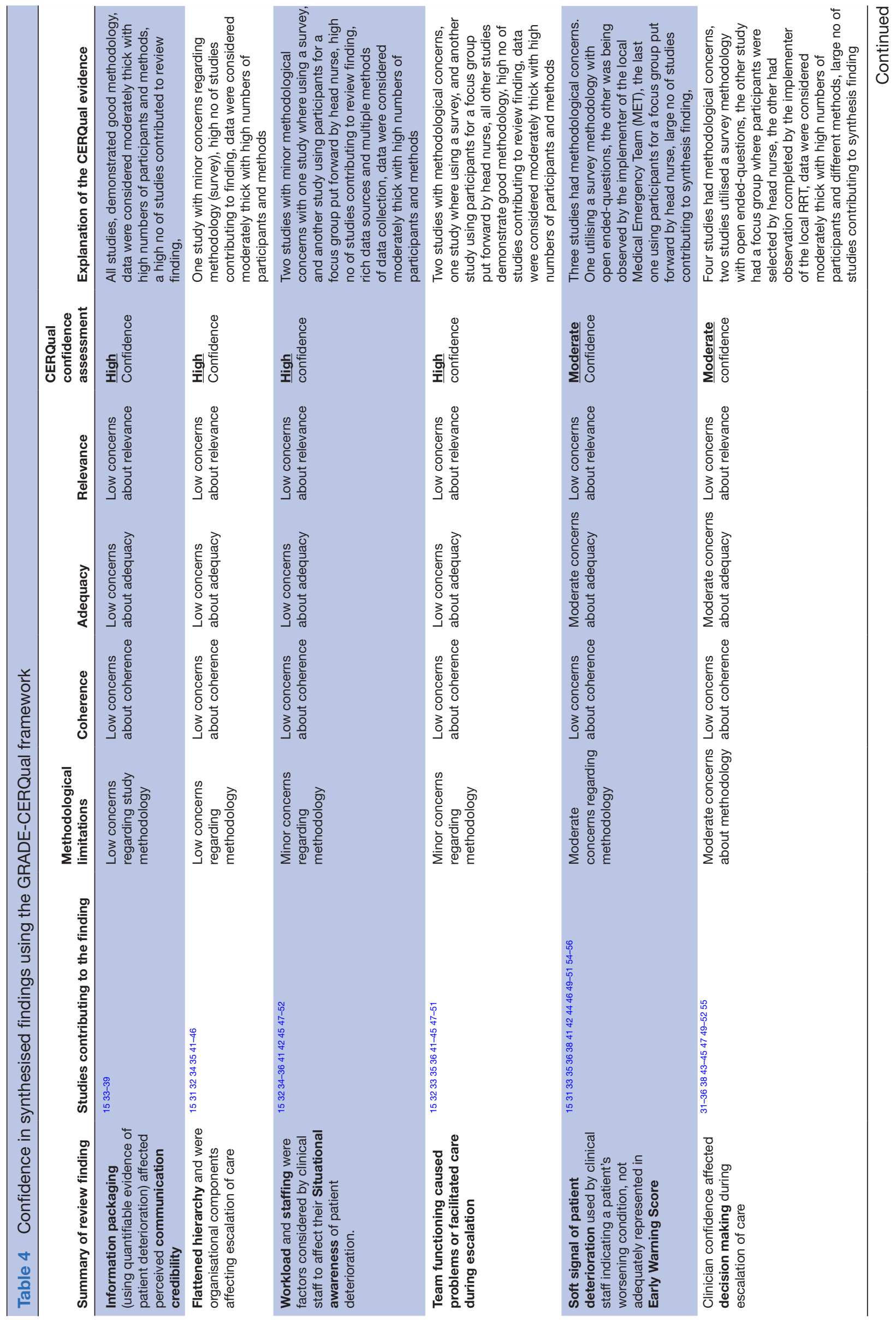


awareness. ${ }^{36} 41424951$ Staff felt that a benchmark 'baseline', meant they could identify any significant changes to patient illness. It was not uncommon for staff to employ workarounds during the periods of system pressure such as escalating to the RRT. This was done (rightly or wrongly) to supplement care escalation when medical support was scarce. ${ }^{1547}$

\section{Team functioning (high GRADE-CERQual evidence)}

Seven studies found poor team relationships were a barrier to escalating patient care, resulting in significant delays. ${ }^{1523335424550}$ Poor team working was presented as tasks being done sequentially rather than concurrently, or where there was a lack of role definition. ${ }^{35} 3641-454748$ Staff believed a lack of understanding of team roles and the care individuals could provide contributed to uncertainty about to whom patients should be escalated. ${ }^{35414445}$ Poor team functioning meant staff felt deterred from escalating care due to negative emotions such as fear of reprimand, fear of being wrong, intimidation and retribution. ${ }^{32} 35424549-5153$ Escalation to outside resources, such as the RRT, was sometimes perceived to be negative ${ }^{154345}$ with staff reporting that they preferred to cope with a patient deterioration.

\section{Soft signals of patient deterioration and EWS (moderate GRADE-CERQual evidence)}

Staff at times overruled the EWS derived escalation pathways using other patient related factors in their decision-making process when considering escalation. ${ }^{15} 31333536384142444649-51$ 54-56 They identified factors additional to standard EWS variables which caused them concern about a patient's condition (see online supplemental file 4). These patient factors or 'soft signals of deterioration', were (from most to least common finding in studies); pale skin, ${ }^{15} 31414950$ respiratory pattern (as distinct from respiratory rate),${ }^{15337}$ blood loss, ${ }^{3649}$ personality change, ${ }^{36}{ }^{49}$ patient complaint, ${ }^{50}{ }^{54}$ skin temperature $^{55}$ and patient fatigue (observed or reported). ${ }^{15}$ Nine studies found patient assessment was integral to detecting the 'soft signals of deterioration' including the early signs of worsening illness before a triggering EWS was evident. 153536383946495055 Two papers described how staff felt that EWS protocols could place barriers to escalation when patients did not meet the trigger threshold but nurses felt they required an increase in care surveillance. ${ }^{3350}$ In some instances staff felt they had to wait for a deterioration to occur before being able to escalate ${ }^{33}$ but in others they continued to escalate despite normal EWS. ${ }^{46} 4951$

\section{Decision making (moderate GRADE-CERQual evidence)}

Escalation decision making involved clinical reasoning surrounding the detection, communication and management of escalation of care. Seven papers found that clinician confidence is a facilitator to decision making during patient deterioration management. ${ }^{31-33} 35364450$ Confidence can be derived from staff providing peer support to 
one another, training or education level. Shared team decisions were sometimes an escalation facilitator. ${ }^{38} 4445474951$ However, a lack of consensus in decision making particularly for end of life care,$^{32}$ was seen as problematic ${ }^{3443495}$ often leading to deviation from guidelines or escalation protocols. Lack of consistency in decisions meant escalation of care demonstrated response variability, ${ }^{34}$ leading to differing and unpredictable priorities. ${ }^{47}$ There was also evidence of clinicians assuming physiology changes were not significant and waiting for confirmation of deterioration before responding meaningfully. ${ }^{43}$

\section{Clinical assessment skills and clinical experience (moderate GRADE-CERQual evidence)}

Clinical assessment involved looking, listening and touching the patient to identify respiratory, skin, neurological or physiological abnormalities. ${ }^{15} 49515557$ The ability to clinically assess patients well enabled staff to make better escalation of care decisions, ${ }^{35} 36384349-5155$ particularly as the ability to detect 'soft signs' was seen as key. Conversely, undertaking a poor patient clinical assessment posed barriers to illness detection. ${ }^{15}$ Many studies found that as staff gained experience of deteriorating patients they were better able to detect and predict impending illness. 15363842434649505455

\section{DISCUSSION}

We identified 24 qualitative studies of moderate to high methodological quality that identify how HF affect escalation of care. Our evidence synthesis has contributed to escalation of care literature and themes derived from analysis are pertinent to clinical practice.

The studies within this synthesis demonstrated that EWS provide staff with a tool that facilitates communication of concerns and assists workload prioritisation. ${ }^{33}$ Studies reported successful escalation of care was best facilitated when a patient's deterioration packaged neatly with quantifiable evidence. However, some staff in the synthesised studies felt able to escalate nontriggering patients requiring medical attention, although this process was acknowledged to be challenging. It was also suggested that some staff can anticipate clinical deterioration before a triggering $\mathrm{EWS}^{58}$ and that there are soft signals (fatigue, skin temp/colour, patient complaint, personality change, blood loss, respiratory pattern), of deterioration recognised by nurses but are not adequately captured by EWS in their current format. Many studies also found that as staff gained experience of deteriorating patients, they were better able to predict deterioration patterns and anticipate problems. It seems that the EWS alone may not maximise improvements to patient outcomes. ${ }^{5960}$ Evidence suggests that organisations should facilitate good patient assessment, as this was key to detecting soft signals that would otherwise go undetected through an alerting system. Research should also aim to identify how clinical staff anticipate problems in certain patient groups and how they recognise and respond to these to ultimately create safety. ${ }^{61}$ It is evident that the literature does not fully report good escalation catches $^{62}$ such as rescued non-triggering sick patients. This event is in effect invisible and not measured in current healthcare evaluation systems or metrics. Incorporating this tacit knowledge into education programmes or simulation training and scenarios, may be a feasible strategy to improve care escalation.

A flattened hierarchy ${ }^{63}$ was implemented in the aviation industry when it was discovered that a number of flight incidents may have been avoided had the copilot been empowered to challenge the pilot. ${ }^{64}$ Synthesised studies identified that a Flattened Hierarchy was felt by healthcare staff to be a positive strategy for escalating care of deteriorating patients (escalation can be initiated from anyone). However, the effectiveness of a flattened hierarchy may be influenced by poor team functioning. Poor team working was a common barrier to escalation of care identified in this evidence synthesis. This finding is corroborated by a retrospective case records review of preventable hospital deaths ${ }^{3}$ and a literature review on FTR following surgery. ${ }^{65}$ In both publications, the authors isolated several contributory factors such as poor team communication, leadership and decision making. Without adequate team communication, the benefits of a flattened hierarchy and team decision making may be lost. If organisations wish to implement a flattened hierarchy escalation system this must also be complemented with an emphasis on non-technical skills and training ${ }^{66}$ before evidence of full patient benefit.

A clinically significant theme to emerge from the synthesised findings was that the greater the workload, the less staff felt they were able to keep track of patient illness or monitor their patients. This sometimes resulted in staff undermonitoring their patients causing some triggering patient deterioration to go unnoticed. ${ }^{50}$ This finding is supported by a recent study demonstrating that lower numbers of registered nurses led to a higher rate of missed vital signs observation. ${ }^{67}$ Organisations could focus on reducing workload, (an unlikely solution), or improving vital signs monitoring processes. A recent option is utilising wearable continuous monitoring that may reduce the nursing workload spent performing regular vital signs observation rounds. ${ }^{68}$

Other significant clinical effects of high workloads may be a reduction in staff ability to detect deterioration in patients who are not triggering, losing the human safety net for false negative (non-triggering) patients. When mental capacity is limited with reduced team resources, this will directly affect an individual's situational awareness of the environment as mental resources reduce as cognitive demands increase. ${ }^{69} \mathrm{~A}$ recent study found the risk of death increased by $3 \%$ for every day a patient experienced nurse staffing levels below ward mean. ${ }^{70}$ Poor situational awareness, reduced ability to detect soft signals of deterioration and undermonitoring may explain these results. Conversely, staff described improved situational awareness when there was continuity to their patient care. 
This was felt to facilitate staff in detecting often nuanced clinical changes or soft signals and also bridged the care elements through a patients illness. ${ }^{71}$ A strong local emphasis on nursing continuity should be encouraged as the evidence suggests that this may improve detection of deterioration and care escalation.

Our study has some limitations. Synthesised studies were assessed for their methodological robustness using the GRADE-CERqual and CASP guidelines. This enabled us to present themes with the highest confidence of good quality evidence first, but results may be limited by the data quality or analysis. within the studies themselves. Publication bis may also affect results that were included. Broadly, studies were methodologically sound but consistently failed to explore the relationship of the researcher to the participants, or this was not explicitly documented. There was also only one study identified that used patients and relatives as study participants. ${ }^{72}$

\section{Conclusion}

This evidence synthesis has identified HF that affect escalation of care. EWS have clinical benefits but can sometime impede escalation in patients not meeting the escalation threshold. Staff use other factors (soft signals) not captured in EWS to escalate care. The literature supports strategies that improve the escalation process such as good patient assessment skills. An organisational emphasis on non-technical skills and team cohesion should be synonymous with a flattened hierarchy to enable effective care escalation.

Twitter Jody Ede @jodyede0x

Contributors All authors have substantially contributed to this work and have agreed the final manuscript. JE designed and led the project. TP provided specialist librarian knowledge and assisted in conducting literature searches. JD, VW, RE and PJW have substantially contributed to this work, provided expertise and have agreed the final manuscript.

Funding JE, Clinical Doctoral Research Fellow, NIHR (NIHR300509) is funded by Health Education England (HEE) / National Institute for Health Research (NIHR) for this research project. PJW is funded by the National Institute of Health Research (NIHR), UK and the NIHR Biomedical Research Centre, Oxford, during the conduct of the study. JD and VW are funded by Health Innovation Challenge Fund (HICFR9-524 and WT-103703/Z/14/Z), a parallel funding partnership between the Department of Health and Wellcome Trust.

Disclaimer The views expressed in this publication are those of the author(s) and not necessarily those of the NIHR, University of Plymouth, NHS or the UK Department of Health and Social Care.

Competing interests PJW report significant grants from the National Institute of Health Research (NIHR), UK and the NIHR Biomedical Research Centre, Oxford, during the conduct of the study. PJW report modest grants and personal fees from Sensyne Health, outside the submitted work. PJW holds shares in the company.

Patient consent for publication Not required

Ethics approval We did not require ethical approval as no primary data were collected.

Provenance and peer review Not commissioned; externally peer reviewed.

Data availability statement Data are available upon request.

Supplemental material This content has been supplied by the author(s). It has not been vetted by BMJ Publishing Group Limited (BMJ) and may not have been peer-reviewed. Any opinions or recommendations discussed are solely those of the author(s) and are not endorsed by BMJ. BMJ disclaims all liability and responsibility arising from any reliance placed on the content. Where the content includes any translated material, BMJ does not warrant the accuracy and reliability of the translations (including but not limited to local regulations, clinical guidelines, terminology, drug names and drug dosages), and is not responsible for any error and/or omissions arising from translation and adaptation or otherwise.

Open access This is an open access article distributed in accordance with the Creative Commons Attribution 4.0 Unported (CC BY 4.0) license, which permits others to copy, redistribute, remix, transform and build upon this work for any purpose, provided the original work is properly cited, a link to the licence is given, and indication of whether changes were made. See: https://creativecommons.org/ licenses/by/4.0/.

\section{ORCID iDs}

Jody Ede http://orcid.org/0000-0001-7289-6991

Ruth Endacott http://orcid.org/0000-0002-4352-4600

Peter J Watkinson http://orcid.org/0000-0003-1023-3927

1 Ghaferi AA, Osborne NH, Birkmeyer JD, et al. Hospital characteristics associated with failure to rescue from complications after pancreatectomy. J Am Coll Surg 2010;211:325-30.

2 Ghaferi AA, Birkmeyer JD, Dimick JB. Hospital volume and failure to rescue with high-risk surgery. Med Care 2011;49:1076-81.

3 Hogan H, Healey F, Neale G, et al. Preventable deaths due to problems in care in English acute hospitals: a retrospective case record review study. BMJ Qual Saf 2012;21:737-45.

4 Donaldson L. An organisation with a memory. Clin Med 2002:2:452-7.

5 NCEPOD. Themes and recommendations common to all Hospital Specialities, 2018.

6 Ede J, Jeffs E, Vollam S, et al. A qualitative exploration of escalation of care in the acute ward setting. Nurs Crit Care 2020;25:171-8.

7 NRLS national patient safety incident reports: commentary 2018.

8 Johnston MJ, Arora S, King D, Bouras G, et al. A systematic review to identify the factors that affect failure to rescue and escalation of care in surgery. Surgery 2015;157:752-63.

9 Findlay G, Shotton H, Mason K. Time to intervene? Natl Confid Enq into Patient Oucome Death 2012:1-125.

10 NHS Improvement. The adult patient who is deteriorating: sharing learning from literature, incident reports and root cause analysis investigations, 2016: 23.

11 Rowan K, Adam S, Ball C. Evaluation of outreach services in critical care, 2004: 399.

12 Johnston M, Arora S, Anderson O, et al. Escalation of care in surgery: a systematic risk assessment to prevent avoidable harm in hospitalized patients. Ann Surg 2015;261:831-8.

13 Mochizuki K, Shintani R, Mori K, et al. Importance of respiratory rate for the prediction of clinical deterioration after emergency department discharge: a single-center, case-control study. Acute Med Surg 2017;4:1-7.

14 Shearer B, Marshall S, Buist MD, et al. What stops Hospital clinical staff from following protocols? an analysis of the incidence and factors behind the failure of bedside clinical staff to activate the rapid response system in a multi-campus Australian metropolitan healthcare service. BMJ Qual Saf 2012;21:569-75.

15 Donohue LA, Endacott R. Track, trigger and teamwork: communication of deterioration in acute medical and surgical wards Intensive Crit Care Nurs 2010;26:10-17.

16 Shamseer L, Moher D, Clarke M. PRISMA-P (Preferred Reporting Items for Systematic review and Meta-Analysis Protocols) 2015 checklist : recommended items to address in a systematic review protocol *. BMJ 2015;349:g7647

17 Stern C, Jordan Z, Mcarthur A. Systematic reviews, step by step developing the review question and inclusion criteria, 2014: 114.

18 Ede J, Westgate V, Petrinic T, et al. How human factors affect escalation of care: a protocol for a qualitative evidence synthesis of studies. BMJ Open 2019;9:e025969.

19 Carayon P, Schoofs Hundt A, Karsh B-T, et al. Work system design for patient safety: the SEIPS model. Qual Saf Health Care 2006;15 Suppl 1:50-8.

20 Lincoln YS, Guba EG. Naturalistic inquiry. Sage Publications, 1985

21 Greenhalgh T. How to read the basics of evidence based medicine, 2001: 315.

22 Tacconelli E. Systematic reviews: CRD's guidance for undertaking reviews in health care, 2010: 10.

23 Lewin S, Booth A, Glenton C, et al. Applying GRADE-CERQual to qualitative evidence synthesis findings: introduction to the series. Implement Sci 2018;13:2.

24 Colvin CJ, Garside R, Wainwright M, et al. Applying GRADECERQual to qualitative evidence synthesis findings-paper 4: how to assess coherence. Implement Sci 2018;13:13. 
25 Booth A, Rashidian A, Noyes J. Applying GRADE-CERQual to qualitative evidence synthesis findings - paper 3 : how to assess methodological limitations. Implement Sci 2018;13.

26 Noyes J, Booth A, Lewin S, et al. Applying GRADE-CERQual to qualitative evidence synthesis findings-paper 6 : how to assess relevance of the data. Implement Sci 2018;13:4.

27 Glenton C, Carlsen B, Lewin S, et al. Applying GRADE-CERQual to qualitative evidence synthesis findings-paper 5: how to assess adequacy of data. Implement Sci 2018;13:14.

28 Lewin S, Bohren M, Rashidian A, et al. Applying GRADE-CERQual to qualitative evidence synthesis findings-paper 2: how to make an overall CERQual assessment of confidence and create a summary of qualitative findings table. Implement Sci 2018;13:10.

29 Booth AA, Noyes J, Flemming K. Guidance on choosing qualitative evidence synthesis methods for use in health technology assessments of complex interventions, 2016. Available: Http// Wwwintegrate-HtaEu/Downloads/

30 Barnett-Page E, Thomas J. Methods for the synthesis of qualitative research: a critical review. BMC Med Res Methodol 2008;9.

31 James J, Butler-Williams C, Hunt J, et al. Vital signs for vital people: an exploratory study into the role of the healthcare assistant in recognising, recording and responding to the acutely ill patient in the general ward setting. J Nurs Manag 2010;18:548-55.

32 Currey J, Allen J, Jones D. Critical care clinician perceptions of factors leading to medical emergency team review. Aust Crit Care 2018;31:87-92.

33 Andrews T, Waterman H. Packaging: a grounded theory of how to report physiological deterioration effectively. J Adv Nurs 2005;52:473-81.

34 Mackintosh N, Rainey H, Sandall J. Understanding how rapid response systems may improve safety for the acutely ill patient: learning from the frontline. BMJ Qual Saf 2012;21:135-44.

35 Johnston M, Arora S, King D, et al. Escalation of care and failure to rescue: a multicenter, multiprofessional qualitative study. Surgery 2014; 155:989-94.

36 Hart PL, Spiva L, Dolly L, et al. Medical-Surgical nurses' experiences as first responders during deterioration events: a qualitative study. $J$ Clin Nurs 2016;25:3241-51.

37 Massey D, Chaboyer W, Anderson V. What factors influence ward nurses' recognition of and response to patient deterioration? an integrative review of the literature. Nurs Open 2017;4:6-23.

38 Searle Leach L, Mayo A. How RNs rescue patients: a qualitative study of RNs' perceived involvement in rapid response teams. Qual Saf Heal Care 2010;19.

39 Mackintosh N, Humphrey C, Sandall J. The habitus of 'rescue' and its significance for implementation of rapid response systems in acute health care. Soc Sci Med 2014;120:233-42.

40 Hart LJ, Carr C, Fullerton JT. Task analysis as a resource for strengthening health systems. J Midwifery Womens Health 2016;61:257-62.

41 Mackintosh N, Watson K, Rance S, et al. Value of a modified early obstetric warning system (MEOWS) in managing maternal complications in the peripartum period: an ethnographic study. BMJ Qual Saf 2014;23:26-34.

42 Brady PW, Goldenhar LM. A qualitative study examining the influences on situation awareness and the identification, mitigation and escalation of recognised patient risk. BMJ Qual Saf 2014:23:153-61.

43 Elmufdi FS, Burton SL, Sahni N, et al. Clinical and sociocultural factors associated with failure to Escalate care of deteriorating patients. Am J Med Qual 2018;33:391-396.

44 Massey D, Chaboyer W, Aitken L. Nurses' perceptions of accessing a medical emergency team: a qualitative study. Aust Crit Care 2014;27:133-8.

45 Astroth KS, Woith WM, Stapleton SJ, et al. Qualitative exploration of nurses' decisions to activate rapid response teams. J Clin Nurs 2013;22:2876-82.

46 Burns KA, Reber T, Theodore K, et al. Enhanced early warning system impact on nursing practice: a phenomenological study. J Adv Nurs 2018;74:1150-6.

47 Martland J, Chamberlain D, Hutton A, et al. Communication and general concern criterion prior to activation of the rapid response team: a grounded theory. Aust Health Rev 2016;40:477-83.

48 Peebles E, Subbe CP, Hughes P, et al. Timing and teamwork--an observational pilot study of patients referred to a Rapid Response
Team with the aim of identifying factors amenable to re-design of a Rapid Response System. Resuscitation 2012;83:782-7.

49 Chua WL, Mackey S, Ng EKC, et al. Front line nurses' experiences with deteriorating ward patients: a qualitative study. Int Nurs Rev 2013;60:501-9.

50 Petersen JA, Rasmussen LS, Rydahl-Hansen S. Barriers and facilitating factors related to use of early warning score among acute care nurses: a qualitative study. BMC Emerg Med 2017:17:36.

51 Braaten JS. Ce: original research: hospital system barriers to rapid response team activation: a cognitive work analysis. Am J Nurs 2015;115:22-32.

52 Chua WL, Legido-Quigley H, Ng PY, et al. Seeing the whole picture in enrolled and registered nurses' experiences in recognizing clinical deterioration in general ward patients: a qualitative study. Int $J$ Nurs Stud 2019;95:56-64.

53 Massey CN, Feig EH, Duque-Serrano L, et al. Well-Being interventions for individuals with diabetes: a systematic review. Diabetes Res Clin Pract 2019;147:118-33.

54 Mohammmed Iddrisu S, Hutchinson AF, Sungkar Y, et al. Nurses' role in recognising and responding to clinical deterioration in surgical patients. J Clin Nurs 2018;27:1920-30.

55 Bunkenborg G, Samuelson K, Åkeson J, et al. Impact of professionalism in nursing on in-hospital bedside monitoring practice. J Adv Nurs 2013;69:1466-77.

56 Foley C, Dowling M. How do nurses use the early warning score in their practice? A case study from an acute medical unit. $J$ Clin Nurs 2019;28:1183-92.

57 Huang H, Chen L, Sanberg PR. Clinical achievements, obstacles, falsehoods, and future directions of cell-based neurorestoratology. Cell Transplant 2012;21 Suppl 1:3-11.

58 Clifton DA, Clifton L, Sandu D-M, et al. 'Errors' and omissions in paper-based early warning scores: the association with changes in vital signs - a database analysis. BMJ Open 2015;5:e007376-7.

$59 \mathrm{Kim}$ WY, Shin YJ, Lee JM, et al. Modified early warning score changes prior to cardiac arrest in general wards. PLOS One 2015;10:e0130523-11.

60 McGaughey J, O'Halloran P, Porter S, et al. Early warning systems and rapid response to the deteriorating patient in hospital: a realist evaluation. J Adv Nurs 2017;73:3119-32.

61 Vincent C. The essentials of patient safety the essentials of patient safety adapted from patient safety. 2nd Edn, 2001.

62 Wallace SC, Mamrol C, Finley E. Promote a culture of safety with good catch reports patient safety analyst data analyst Pennsylvania patient safety authority corresponding author. 14, 2017.

63 HSE. High reliability organisations high reliability organisations a review of the literature, 2011.

64 Green B, Oeppen RS, Smith DW, et al. Challenging hierarchy in healthcare teams - ways to flatten gradients to improve teamwork and patient care. Br J Oral Maxillofac Surg 2017:55:449-53.

65 Ghaferi AA, Dimick JB. Importance of teamwork, communication and culture on failure-to-rescue in the elderly. Br J Surg 2016;103:e47-51.

66 Yule S, Flin R, Paterson-Brown S, et al. Non-Technical skills for surgeons in the operating room: a review of the literature. Surgery 2006;139:140-9.

67 Redfern OC, Griffiths P, Maruotti A, et al. The association between nurse staffing levels and the timeliness of vital signs monitoring: a retrospective observational study in the UK. BMJ Open 2019:9:e032157.

68 Areia C, Young L, Vollam S, et al. Wearability testing of ambulatory vital sign monitoring devices: prospective observational cohort study. JMIR Mhealth Uhealth 2020;8:e20214.

69 Macdonald JSP, Lavie N. Visual perceptual load induces inattentional deafness. Atten Percept Psychophys 2011;73:1780-9.

70 Griffiths P, Maruotti A, Recio Saucedo A, et al. Nurse staffing, nursing assistants and hospital mortality: retrospective longitudinal cohort study. BMJ Qual Saf 2019;28:609-17.

71 Haggerty JL, Reid RJ, Freeman GK, et al. Continuity of care: a multidisciplinary review. BMJ 2003;327:1219-21.

72 King L, Peacock G, Crotty M, et al. Consumers' perspectives on their involvement in recognizing and responding to patient deteriorationDeveloping a model for consumer reporting. Health Expect 2019;22:385-95.

73 Endsley MR. Design and evaluation for situation awareness enhancement. Proc Hum Factors Soc Annu Meet 1988:32:97-101. 